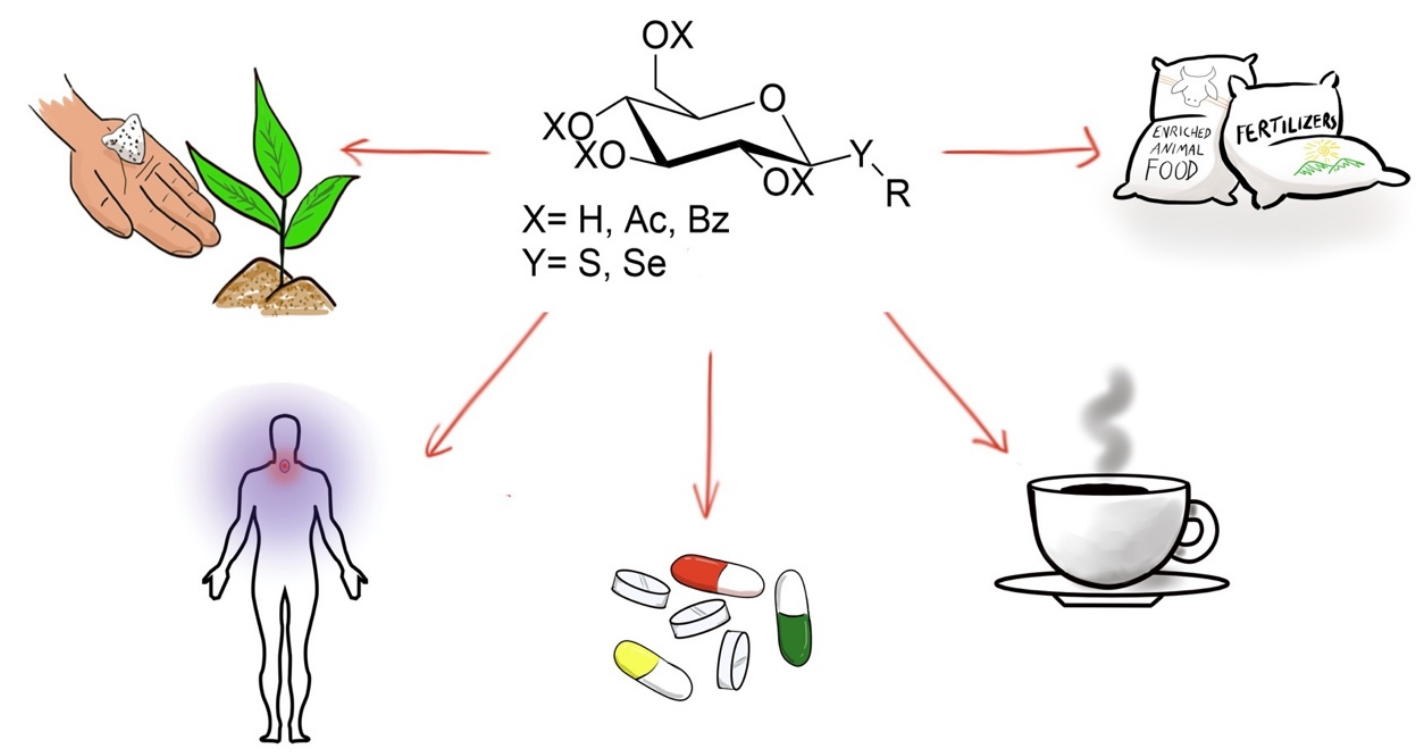

Structural diversity and potential applications of chalcogen sugars.

\title{
CALCOGENO-AÇÚCARES: PERSPECTIVAS, SÍNTESES E APLICAÇÕES
}

Beatriz F. dos Santos ${ }^{1 *}$, Samuel L. Silva ${ }^{1}$, Alcindo A. Dos Santos ${ }^{1}$

1- Instituto de Química, Universidade de São Paulo (USP), São Paulo, SP

*e-mail: beatrizfuzinato@usp.br

Resumo: Os carboidratos estão entre as macromoléculas mais abundantes da Terra e têm um amplo espectro de aplicação em síntese orgânica. Análogos de açúcar que contêm modificações estruturais em suas moléculas são de grande interesse científico e vêm ganhando ampla atenção em estudos biológicos e farmacológicos. Modificações envolvendo átomos de calcogênios são particularmente interessantes porque combinam a absorção de glicosídeos no corpo humano com as propriedades desejadas. As modificações químicas feitas na posição C-1 de açúcares são destacadamente importantes devido à alta reatividade desta posição e à estabilidade da ligação anomérica calcogenocarbono.

Palavras-chave: açúcares, carboidratos, calcogênios

\section{Chalcogen-sugar's: perspectives, synthesis and applications}

Abstract: Carbohydrates are the most abundant macromolecules on Earth and have a broad spectrum of application in organic synthesis. Sugar analogues that contain structural modifications in their molecule are of great interest in science and have been gaining attention in a wide area of biological and pharmacological studies. Modifications involving chalcogen atoms are particularly interesting because they combine the absorption of glycosides in the human body with desired properties. Modifications made in position C-1 are highlighted due to the high reactivity of this position and the stability of the anomeric chalcogen-carbon bond.

Keywords: sugars, carbohydrates, chalcogens 


\section{Introdução}

A substituição de um grupo hidroxila, de um monossacarídeo por um átomo com propriedades distintas, como o enxofre, selênio, ou telúrio, altera a basicidade, polaridade e solubilidade dos açúcares, além das suas capacidades de estabelecerem ligações de hidrogênio. Após a inserção deste átomo de calcogênio também é possível realizar reações subsequentes para adição de outras porções para conferirem, reunidas, propriedades desejadas. Porém, a substituição de apenas um grupo hidroxila não é uma tarefa trivial, sendo necessários inúmeros passos que envolvem, ou não, a proteção de grupos, seletivamente. ${ }^{1,2}$

Dentre todas as hidroxilas presentes em moléculas de sacarídeos, a hidroxila ligada ao átomo de carbono cetal é particularmente interessante. Este átomo de carbono é anomérico, sendo que quando o açúcar se encontra na sua forma aberta este carbono é carbonílico, e quando está na forma fechada, este carbono se torna um centro quiral, o hidrogênio a ele ligado apresenta $p \mathrm{Ka}$ menor que dos outros e, por consequência maior reatividade. Além disso, as ligações chamadas glicosídicas em geral são feitas, principalmente nesta posição.

Os derivados 1-tioglicosídicos têm sido considerados compostos miméticos de $O$ glicosídeos biologicamente importantes ${ }^{3}$ e, além disso, os $S$-glicosídeos são mais estáveis a degradações química e enzimática em comparação aos análogos de oxigenados. ${ }^{4,5}$ Além disso, a porção tioaçúcar é encontrada em vários medicamentos, produtos naturais e agentes biologicamente ativos, incluindo o inibidor de hSGLT1 (pertencente à classe de inibidores do diabetes do tipo 1), ligante da proteína lectina $\mathrm{A}$, bem como inibidores das enzimas galactosidase e glicosidase (figura 1). $6,7,8,9$ 
Figura 1. Moléculas bioativas contendo o núcleo tioaçúcar.

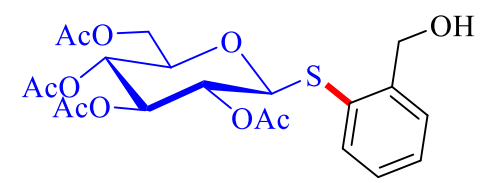

inibidor de hSGLT1

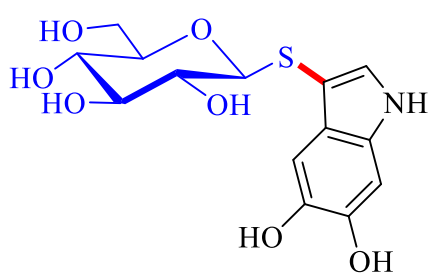

Precursor de polímero de Eumelanina glicosilado

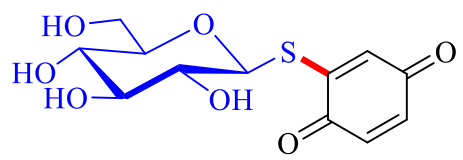

Inibidor de glicosídeo irreversível

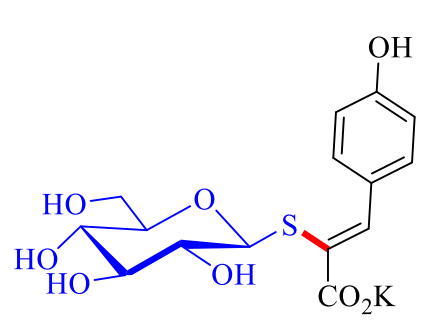

Inibidor de $\beta$-glucosidase de fechamento de folha

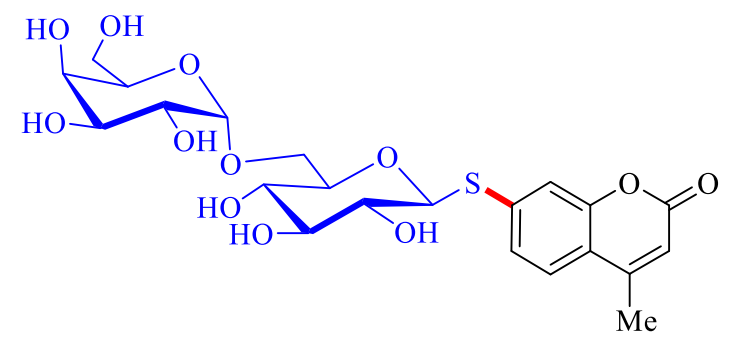

Ligante da Lectina A

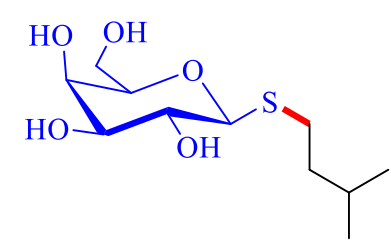

inibidor de $\beta$-galactosidase

Ainda no tocante aos calcogênios, compostos de selênio já foram identificados como metabolitos de bactérias e micro-organismos, ${ }^{10}$ e desde a primeira síntese reportada de um sacarídeo contendo selênio em 1945, diversos avanços foram feitos. Em 1997 o primeiro selenoaçúcar, de fonte natural, foi identificado na planta Astragalus racernosus ${ }^{11}$ e pesquisas recentes identificaram 3 glicosídeos contendo selênio na urina humana. ${ }^{12}$ Do ponto de vista sintético, diversos compostos de selênio são de interesse reacional, pois podem apresentar caráter nucleofílico ou eletrofílico ${ }^{13} \mathrm{e}$ servem como precursores de outras moléculas derivadas de carboidratos, tais como 2deoxiglicosídeos e orto-ésteres, glicais funcionalizados, $C$-glicosídeos, ou mesmo em glicosilações controladas de proteínas. Além disso sabendo do papel do selênio no organismo, pode-se inferir que a síntese destas moléculas é de grande interesse para a produção de suplementos à base de selênio com papel coadjuvante na prevenção de diversas doenças. ${ }^{12}$

Tendo em vista a importância sintética e biológica dos açúcares substituídos na posição anomérica, esta mini revisão visa destacar alguns conceitos e trazer breves relatos da literatura para a síntese e a funcionalização de 1-calcogenoaçúcares de estruturas diversas. 


\section{Síntese de 1-calcogenoaçúcares}

\section{Sintese de 1-tioaçúcares}

Diversas metodologias já foram reportadas na literatura, visando a síntese de 1-tioaçúcares. No geral, os métodos para a síntese de 1-tioaçúcares envolvem o tratamento do glicosil halogenado (building block estratégico) com uma espécie de enxofre nucleofílica (tioureia, dissulfeto de carbono ou tioacetato de potássio) em acetona, dando origem ao sal de tiourônio. ${ }^{14,15,16,17}$ Em seguida, o grupo de enxofre livre $(\mathrm{SH})$ é gerado através de uma hidrólise do sal de tiourônio (esquema 1). Outras metodologias para a obtenção de 1-tioaçúcares incluem o uso de ácidos de Lewis $^{18}$ e a redução de Birch do derivado tiobenzil anomérico. ${ }^{19}$

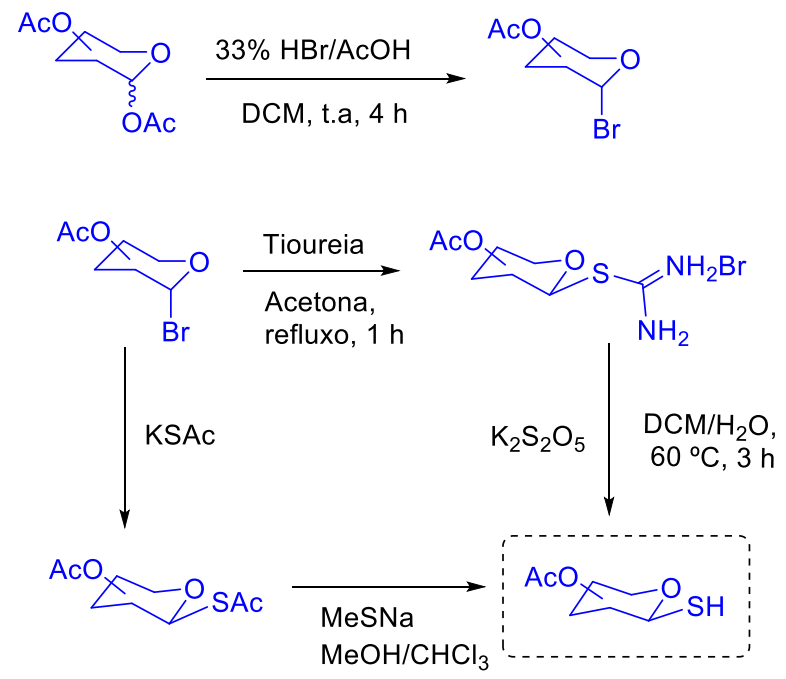

Esquema 1. Estratégias sintéticas gerais de 1-tioaçúcares.

Síntese de Tioglicosídeos Catalisada por Ácidos de Lewis

Como os tioglicosídeos são intermediários versáteis e valiosos na síntese orgânica, ${ }^{20}$ pesquisadores têm se esforçado na síntese e avaliações biológicas desses glicomiméticos. ${ }^{21}$ No entanto, poucos métodos para a síntese de tiossacarídeos foram relatados na literatura até o presente momento. Normalmente, os derivados de tioglicosídeos são mais comumente preparados pela reação do tiofenol com o glicosil per- $O$-acetilado na presença de um ácido de Lewis ${ }^{22,23,24}$ ou pela substituição do átomo de halogênio no carbono anomérico do açúcar por um ânion tiolato. ${ }^{25,26}$ Deste modo, Abbineni e coautores, em $2003^{23}$ relataram um método simples e eficiente para a síntese de tioglicosídeos catalisada por cloreto de índio (III). As reações conduzidas pelos autores foram 
realizadas usando várias glicopiranoses per- $O$-acetiladas como substratos, além de diversos tiois (3 equiv), $\mathrm{MeCN}$ como solvente $\left(2 \mathrm{~mL}\right.$ ), $\mathrm{InCl}_{3}$ (20 ou $40 \mathrm{~mol} \%$ ) e $\mathrm{TiCl}_{4}$ como co-ativador (esquema 2).

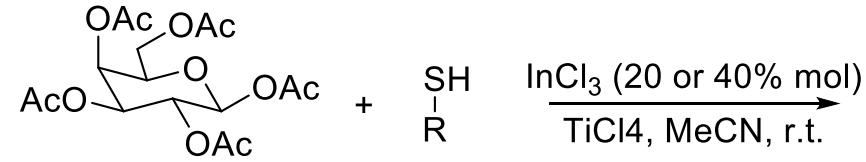

$R=p-$ Toluil, Fenil, Etil

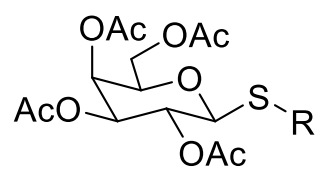

$24-98 \%$

Esquema 2. Síntese de tioglicosídeos, catalisada por $\mathrm{InCl}_{3}$ e $\mathrm{TiCl}_{4}$.

Em 2005, Misra e colaboradores realizaram a síntese one-pot de tioglicosídeos per-Oacetilados a partir de açúcares desprotegidos, utilizando $\mathrm{BF}_{3}$. $\mathrm{OEt}_{2}$ como catalisador (esquema 3). Os autores reportaram a utilização de quantidade estequiométrica de $\mathrm{Ac}_{2} \mathrm{O}$, excesso de $\mathrm{BF}_{3} . \mathrm{OEt}_{2}$ (1,5 equiv) como catalisador, além de diversos açúcares desprotegidos e variados alquil- e aril-tióis. O progresso da reação foi monitorado por cromatografia em camada delgada. Os produtos foram obtidos em rendimentos de 70 a $90 \% .^{24}$
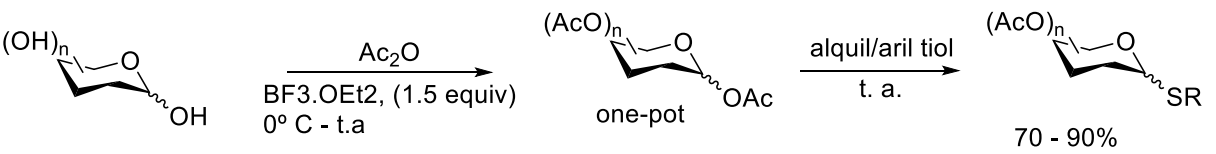

$\mathrm{R}=\mathrm{Etil}, \mathrm{Ph}, \mathrm{p}-\mathrm{MePh}$

Esquema 3. Síntese de tioglicosídeos catalisada por $\mathrm{BF}_{3} . \mathrm{OEt}_{2}$

Síntese de Derivados do 1-Tioaçúcar Catalisada por Paládio

Apesar de serem relativamente simples, as metodologias utilizando ácidos de Lewis são restritas ao escopo do substrato com tiofenóis e sofrem com o uso de reagentes ácidos agressivos. ${ }^{3}$ Assim, o desenvolvimento de protocolos eficientes e seletivos para acessar os derivados de tioaçúcar ainda é desejável. Algumas metodologias já foram descritas na literatura para a 
preparação de derivados de tioaçúcar por meio de reações de acoplamento cruzado C-S, porém essas abordagens são bem menos comuns. ${ }^{27}$

Alami e colaboradores ${ }^{3}$, em 2013 publicaram um trabalho de acoplamento cruzado C-S para a síntese de derivados tioglicosídeos utilizando (hetero) haletos de arila, diversos tioaçúcares e $\mathrm{Pd}(\mathrm{OAc})_{2} / \mathrm{Xantphos} \mathrm{como} \mathrm{catalisador} \mathrm{(esquema} \mathrm{4).} \mathrm{Além} \mathrm{disso,} \mathrm{os} \mathrm{autores} \mathrm{também} \mathrm{fizeram} \mathrm{o} \mathrm{uso}$ de $\mathrm{Et}_{3} \mathrm{~N}$ como base e dioxano como solvente. Os produtos foram obtidos após 1 hora de reação a $100{ }^{\circ} \mathrm{C}$, com rendimentos variando de 61 a $99 \%$.
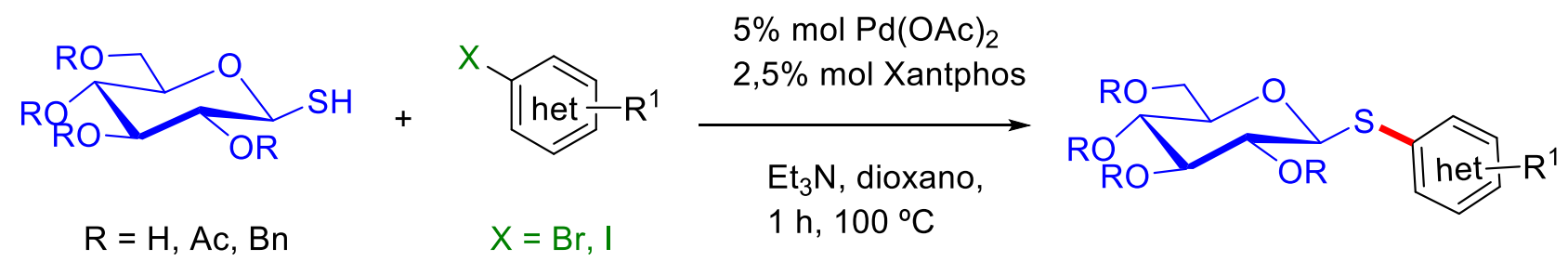

$61-99 \%$

$\mathrm{R}_{1}=4-\mathrm{OMe}, 4-\mathrm{NH}_{2}$, 3-OTBS-4-OMe, 3-OH, 2-OMe, 2-Me, 4- $\mathrm{NO}_{2}$, 4-CHO, 4- $\mathrm{CO}_{2} \mathrm{Et}$, 4-COMe, 3-Br, 4-- $\mathrm{CF}_{3}$

Esquema 4. Síntese de derivados tioglicosídeos utilizando (hetero) haletos de arila

Em 2017, Messaoudi e colaboradores ${ }^{21}$ relataram a síntese de tioglicosídeos derivados do 2-iodoglical utilizando como catalisador o Pd G3-Xantphos. Os autores realizaram uma triagem de solvente, temperatura e tempo reacional, e ao final estabeleceram as melhores condições, sendo estas de: $4 \%$ mol de catalisador, $\mathrm{Et}_{3} \mathrm{~N}$ como base e dioxano como solvente, em um tempo reacional de 2 horas à $60^{\circ} \mathrm{C}$. Os autores obtiveram 17 compostos com rendimentos variando de 18 a $98 \%$ (esquema 5).

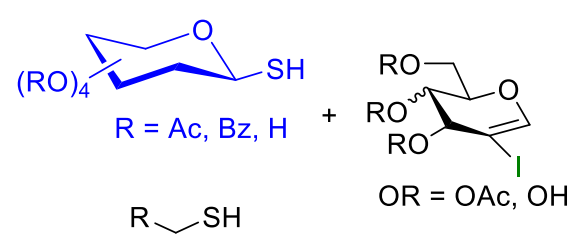

$$
\begin{aligned}
& \text { Pd G3-Xantphos } \\
& \begin{array}{l}
4 \% \mathrm{~mol} \\
\text { Et3N, dioxano, } \\
60^{\circ} \mathrm{C}, 2 \mathrm{~h}
\end{array}
\end{aligned}
$$

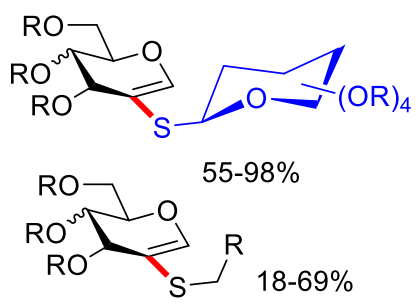

Esquema 5. Síntese de tioglicosídeos derivados do 2-iodoglical. 
Recentemente (em 2019), Messaoudi e colaboradores ${ }^{28}$ propuseram novamente a funcionalização de 1-tioaçúcares, porém desta vez, utilizando uma metodologia de catálise dual fotoredox, empregando um catalisador de níquel (II) e rutênio bipiridina como fotocatalisador (esquema 6). Os autores fizeram o uso de diversos tioaçúcares mono e dissacarídeos, além de diferentes aglicanas, por irradiação com LEDs azuis, DMF como solvente em um tempo reacional de 24 horas à temperatura ambiente. Um aspecto importante desta metodologia foi a utilização de um agente HAT (Hydrogen Atom Transfer) e a ausência de base. Os autores obtiveram 32 compostos em rendimentos de 30 à $96 \%$.
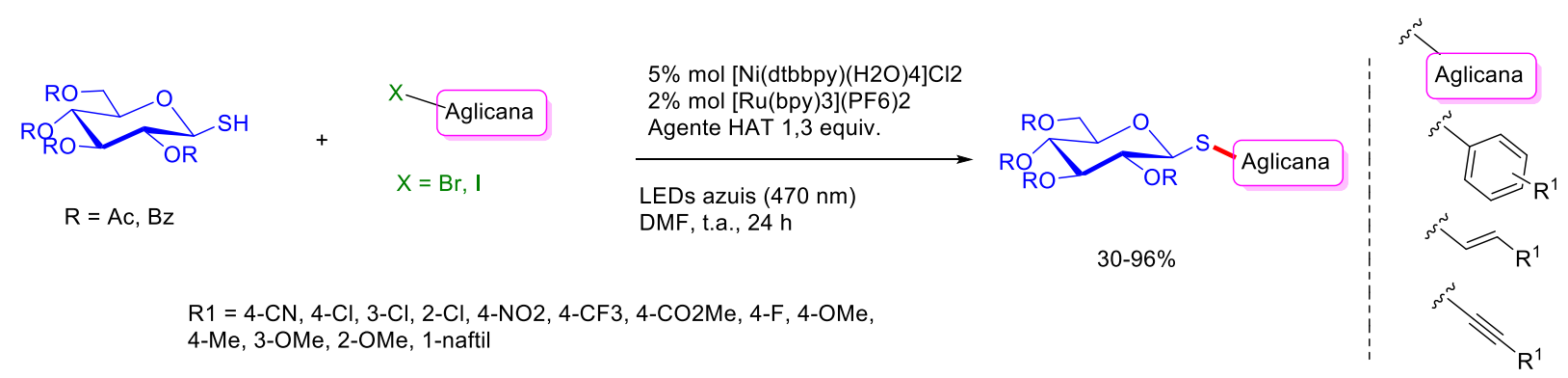

Esquema 6. Funcionalização de 1-tioaçúcares fotocatalisada por níquel

Sintese de tioglicosídeos utilizando cloreto de 2-cloro-1,3-dimetilimidazol

Shoda e colaboradores relataram uma nova abordagem utilizando o cloreto de 2-cloro-1,3dimetilimidazol (DMC) como catalisador de reações centradas no carbono anomérico de carboidratos sem proteção, sendo este reagente utilizado, entre outras aplicações, para síntese de 1tioaçúcares. Desde então, sínteses de carboidratos sem o uso de grupos protetores têm sido reportadas na literatura, utilizando o DMC como agente ativante do carbono anomérico. ${ }^{1,2}$ Este processo apresenta a vantagem de não requerer etapas de proteção/desproteção, que inerentemente são mais práticos, rápidos e atrativos do ponto de vista econômico/ambiental. No esquema 7 , está apresentado de maneira genérica, a aplicação do DMC para a preparação de diferentes tioaçúcares. $^{29}$ 

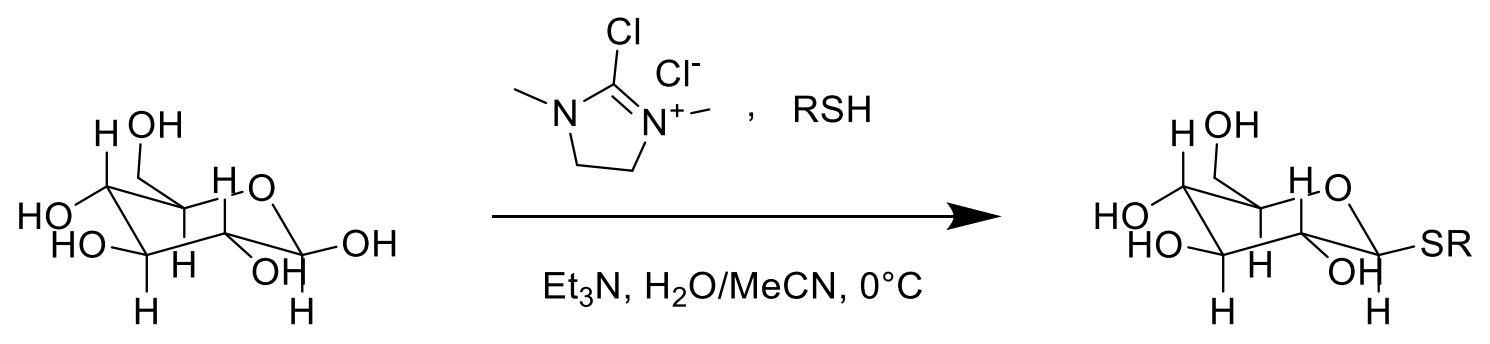

Esquema 7. Síntese de tioaçúcares por catalise com DMC sem proteção dos grupos hidroxila.

\section{Sintese de 1-selenoaçúcares}

A estabilidade de compostos contendo selênio na posição 1 dos carboidratos substituídos ainda é um impedimento para aplicações preparativas desses compostos. Dentre as metodologias desenvolvidas, as mais utilizadas estão centradas no uso de selenoureia para adição de selênio e posterior alquilação, conforme esquema $8 .{ }^{12,30}$

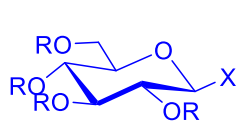

$\mathrm{R}=\mathrm{Ac}, \mathrm{H}$

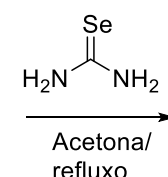

refluxo

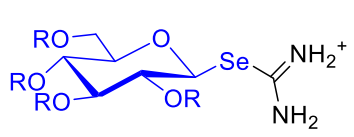

$\mathrm{NH}_{2}$

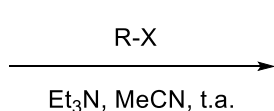

$\mathrm{Et}_{3} \mathrm{~N}, \mathrm{MeCN}, \mathrm{t} . \mathrm{a}$.

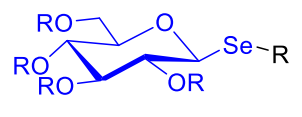

Esquema 8. Síntese de selenoaçúcares utilizando selenoureia como nucleófilos de selênio.

\section{Conclusões}

A presente mini revisão destacou brevemente algumas das possibilidades de síntese de carboidratos contendo átomos de calcogênios, bem como metodologias para a obtenção de 1calcogenoaçúcares funcionalizados. Esta classe de moléculas é de grande interesse biológico e sintético, além disso, apresenta uma grande versatilidade de métodos de preparação de derivados glicosídeos, não se limitando somente às reações mostradas aqui. Apesar de haver um número razoável de exemplos de metodologias fundamentadas na funcionalização do carbono anomérico com resíduos de enxofre, existe um número muito menor de metodologias robustas e reprodutíveis envolvendo compostos de selênio e ainda mais raros são os exemplos com o homólogo, telúrio. Não apenas pela escassez de exemplos com estes outros derivados de calcogênios, mas especialmente pela peculiar atividade que estes podem conferir aos compostos de que fazem parte estrutural, este é 
um ramo da química preparativa de açúcares que representa uma janela de oportunidades, unindo, a importância dos glicosídeos com o perfil redox único dos organocalcogenetos.

\section{Agradecimentos}

FAPESP, Capes, USP.

\section{Referências}

1. Meguro, Y.; Noguchi, M.; Li, G.; Shoda, S. I.; Org. Lett. 2018, $20,76$.

2. Fairbanks, A. J.; Carbohydr. Res. 2021, 499.

3. Brachet, E., Brion, J.-D., Messaoudi, S.; Alami, M.; Adv. Synth. Catal. 2013, 355, 477.

4. Rye, C. S.; Withers, S. G.; Carbohydr. Res. 2004, 339, 699.

5. Metaferia, B. B.; Fetterolf, B. J.; Shazad-ul-Hussan, S.; Moravec, M.; Smith, J. A.; Ray, S.; Gutierrez-Lugo, M.-T.; Bewley, C. A.; J. Med. Chem. 2007, 50, 6326.

6. Castaneda, F.; Burse, A.; Boland, W.; Kinne, R. K-H.; Int. J. Med. Sci. 2007, 4, 131.

7. Rodrigue, J.; Ganne, G.; Blanchard, B.; Saucier, C.; Giguère, D.; Shiao, T. C.; Varrot, A.; Imberty, A.; Roy, R.; Org. Biomol. Chem. 2013, 11, 6906.

8. Elgemeie, G. H.; Farag, A. B.; Amin, K. M.; El-Badry, O. M.; Hassan, G. S.; Med. Chem. 2014, 4, 814.

9. $\quad$ Kato, E.; Nagano, H.; Yamamura, S.; Ueda, M.; Tetrahedron 2003, 59, 5909.

10. Heider, J.; Böck, A.; Adv. Microb. Physiol. 1993, 35, 71.

11. Witczak, Z. J.; Czernecki, S.; Adv. Carbohydr. Chem. Biochem. 1998, 53, 143.

12. Ashok, A.; Zita, T.; Kövér, K. E.; Szilágyi, L.; Carbohydr. Res. 2012, 360, 8.

13. Braga, A. L.; Lütke, D. S.; Vargas, F.; Braga, R. C.; Synlett 2006, 1453.

14. Lee, Y. C.; Stowell, C. P.; Krantz, M. J.; Biochemistry 1976, 15, 3956.

15. Lee, Y. C.; Stowell, C. P., Methods Enzymol. 1982, 83, 278.

16. MacDougall, J. M.; Zhang, X. D.; Polgar, W. E.; Khroyan, T. V.; Toll, L.; Cashman, J. R.; J. Med. Chem. 2004, 47, 5809.

17. Johnston, B. D.; Pinto, B. M.; J. Org. Chem. 2000, 65, 4607.

18. Ibatulin, F. M.; Shabalin, K. A.; JKnis, J. V.; Shavva, A. G.; Tetrahedron Lett., 1998, 39, 7961.

19. Holick, S. A.; Anderson, L.; Carbohydr. Res., 1974, 34, 208.

20. Codée, J. D. C.; Litjens, R. E. J. N.; J. van den Bos, L.; Overkleeft, H. S.; A. van der Marel, G.; Chem. Soc. Rev. 2005, 34, 769.

21. AL-Shuaeeb, R. A. A.; Montoir, D.; Alami, M.; Messaoudi, S. J. Org. Chem. 2017, 82, 6720 .

22. Nicolaou, K. C.; Randall, J. L.; Furst, G. T. J. Am. Chem. Soc. 1985, 107, 5556.

23. Das, S. K.; Roy, J.; Reddy, K. A.; Abbineni, C. Carbohydr. Res. 2003, 338, 2237.

24. Agnihotri, G.; Tiwari, P.; Misra, A. K.; Carbohydr. Res. 2005, 340, 1393.

25. Blanc-Muesser, M.; Defaye, J.; Driguez, H.; Carbohydr. Res. 1978, 67, 305.

26. Apparu, M.; Blanc-Muesser, M.; Defaye, J.; Driguez, H.; Can. J. Chem. 1981, 59, 314.

27. Ibrahim, N.; Alami, M.; Messaoudi, S.; Asian J. Org. Chem. 2018, 7, 2026.

28. Zhu, M.; Dagousset, G.; Alami, M.; Magnier, E.; Messaoudi, S. Org. Lett. 2019, 21, 5132.

29. Li, G.; Dao, Y.; Mo, J.; Dong, S.; Shoda, S.; Ye, X. 2021, 2316.

30. Tiwari, P.; Misra, A. K. ;Tetrahedron Lett. 2006, 47, 2345. 
